\title{
A One-Body Fix
}

\section{from D. H. Sadler and W. A. Scott}

IN U.S. Naval Institute Proceedings for June 1957 (page 680), Captain P. V. H. Weems and Captain R. E. Jasperson outline a method for finding a position by observing one celestial body only. The principle of the method is to use the observed rate of change of altitude, together with the observed altitude itself, to solve directly for latitude and hour angle, and thus to obtain the position. The authors point out that the parallactic angle, $C$ (the angle at the observed body), in the usual navigational spherical triangle is obtainable from:

$\sin C=\sec$ (declination) $\times$ rate of change of altitude with hour angle.

Assuming that the rate of change can be observed with modern techniques to requisite accuracy, they then suggest that tables be prepared to give latitude and hour angle with the three arguments: declination, altitude and parallactic angle, $C$; a specimen page of such a table is illustrated. Interpolation to full accuracy is required for both latitude and hour angle, and variations are given to allow for this; however, the illustrative example gives a false impression of the accuracy obtainable by such tables. It is well known that the second-order terms, which have to be neglected, may amount to more than $\mathrm{I}^{\prime}$ when interpolation is made for all three arguments at intervals of one degree; in H.O. 2 I 4 (H.D. 486) interpolation is usually restricted to declination, which is given at intervals of half a degree. Moreover, the variations are given to two decimals only and their roundings may each contribute errors of $0: 2$.

The table, as suggested, is the same in principle as H.O. 214 , but with the 'azimuth' given to 0 ! 1 instead of $0: 1$, and variations provided for all three arguments; it is simply a tabular solution of a spherical triangle in which two sides (altitude and declination) and the included (parallactic) angle are given. The user could have been saved some work by using the rate of change of altitude as argument directly.

But, the crucial question is whether this rate of change can be measured sufficiently accurately, even by the elaborate facilities and equipment on board the U.S.S. Compass Island, the U.S. Navy's specially fitted navigational ship with a stabilized platform on a 67 -ton tower. (For a brief description see U.S. Naval Institute Proceedings for February 1957, page 232.) The accuracy required depends on the altitude; at the zenith a position can be determined from the observed altitude only, but the error in position can rise to as large as the error in the parallactic angle itself. Thus an error of 0 : I in the difference in altitude over a period of 4 minutes of time may give rise to an error in position of six miles, even when $C$ is small; when the parallactic angle, $C$, is near $90^{\circ}$ the method breaks down completely since the angle cannot be determined from its sine. For the accuracy of $0 ?_{1}$ in position considered by the authors, either the consistency of measurement of altitude (and thus of stability) must be substantially increased or the time-interval greatly lengthened. In the latter case complexities will arise because of the true motion of the body observed, the motion of the ship and the fact that the rate of change of altitude is itself not constant over any appreciable time-interval.

The authors rather deride the use of observed azimuth, but if there is available a 
platform stabilized to the accuracy considered then it should be possible to observe the azimuth more accurately and more easily than the rate of change of altitude. The position is then determined by the solution of a spherical triangle in which two sides (altitude and declination) and the angle opposite one (the azimuth) are known; there are many practical methods of doing this and, in fact, a mechanical or electronic solution must be regarded as simple compared to the difficulty of stabilization and automatic observation.

\title{
The Accuracy of Astronomical Observations at Sea
}

\author{
from Commander J. M. Sharpey-Schafer, R.N.
}

THE statistical analysis in the Institute Working Party's report on the accuracy of astronomical observations at sea (this Journal, 10, 223 ) is a fine piece of work for which we should all be most grateful to Mr. Sadler and the staff of H.M. Nautical Almanac Office. Indeed it is perhaps not out of place to recall the traditions by which the Royal Observatory supported by the Government of the day has always helped navigation, and to express gratitude to the Astronomer Royal for permitting and encouraging the work.

One or two quite, interesting questions arise. There is a clear pattern in the observations of 50 per cent taken to $0: 2,25$ per cent to $0: 5$ and 25 per cent to whole I'. The table 'Accuracy of Sextant reading', 'Observers' Estimates', Table VIII and, more important, inspection of the forms, refer. Several errors given as 'Nil' on the forms seemed to indicate that within a whole I' was good enough. If all the observations had been taken to a whole $I^{\prime}$ or all had been taken to $0: 2$, it does not seem beyond the bounds of possibility that different answers would have been achieved. In the analysis they have not been split up. In that particular respect the analysis does not show that you might do better if you tried harder, although later it does analyse several observers who took a large number of sights and selects the better observers.

The next point is the number of shots. The report states that 'as far as the present observations are concerned the errors of a single shot appear to be just as accurate as those of the mean of 2 or 3'. Mr. Sadler has explained to me that this statement drawn from Table I, is given to explain why they are combined and subsequent precedures. Furthermore the report says 'practically all the observations based on the mean of 2 or 3 shots were made by only 2 observers'. Section 6 also refers. It seems important to emphasize that it is not a major conclusion of the analysis, indeed it appears to clash rather considerably with the methods of observation in surveying, astronomy and many other things; so much so that it just raises a slight doubt as to whether something has crept into the whole trial which might be swamping the results. However, what is clear is that the Working Party in its future work ought to test the subject of the number of shots from $1-7$ against high and low-power magnification in a different way, which might be done simply by a few observers on shore in a day.

Similarly a minor point is the State of the Sea not showing any significance, 